Published in final edited form as:

Genet Epidemiol. 2019 July ; 43(5): 522-531. doi:10.1002/gepi.22201.

\title{
A simple approximation to bias in the genetic effect estimates when multiple disease states share a clinical diagnosis
}

\author{
Iryna Lobach ${ }^{1,}$, Inyoung Kim ${ }^{2}$, Alexander Alekseyenko ${ }^{3}$, Siarhei Lobach ${ }^{4}$, and Li Zhang ${ }^{5}$ \\ ${ }^{1}$ Department of Epidemiology and Biostatistics, University of California, San Francisco, San \\ Francisco, USA \\ 2Department of Statistics, Virginia Tech University, Blacksburg, VA, USA \\ ${ }^{3}$ Department of Public Health Sciences, Medical University of South Carolina, Charleston, SC, \\ USA \\ ${ }^{4}$ Applied Mathematics and Computer Science Department, Belarusian State University, Minsk, \\ Belarus \\ ${ }^{5}$ Department of Medicine, University of California, San Francisco, San Francisco, USA
}

\begin{abstract}
Case-control genome-wide association (CC-GWAS) studies might provide valuable clues to the underlying pathophysiologic mechanisms of complex diseases, such as neurodegenerative disease, cancer. A commonly overlooked complication is that multiple distinct disease states might present with the same set of symptoms and hence share a clinical diagnosis. These disease states can only be distinguished based on a biomarker evaluation that might not be feasible in the whole set of cases in the large number of samples that are typically needed for CC-GWAS. Instead, the biomarkers are measured on a subset of cases. Or an external reliability study estimates the frequencies of the disease states of interest within the clinically diagnosed set of cases. These frequencies often vary by the genetic and/or non-genetic variables. We derive a simple approximation that relates the genetic effect estimates obtained in a traditional logistic regression model with the clinical diagnosis as the outcome variable to the genetic effect estimates in the relationship to the true disease state of interest. We performed simulation studies to assess accuracy of the approximation that we've derived. We next applied the derived approximation to the analysis of the genetic basis of innate immune system of Alzheimer's disease.
\end{abstract}

\section{Keywords}

bias; Alzheimer's disease; Misclassifiaction of disease status; Kullback-Leibler; divergence

"Corresponding author: Iryna Lobach, Ph.D., Division of Biostatistics, Department of Epidemiology and Biostatistics, University of California, San Francisco, Iryna.lobach@ucsf.edu, Phone: 415-476-6115. 


\section{INTRODUCTION}

Case-control genome-wide analyses scan (CC-GWAS) is a tool that is widely used to elucidate the genetic basis of complex diseases. A common complication is that multiple distinct disease states share the observed symptoms and hence the clinical diagnosis. Frequencies of the disease states within the clinical diagnosis often vary by the key variables. If the disease states have distinct genetic basses, the analyses with a clinical diagnosis as an outcome variable might be substantially biased (Carroll et al, 2006).

The specific example that motivated this study is the analyses of the genetic susceptibility to Alzheimer's disease (AD). The clinical diagnosis of AD is typically made based on a set of descriptive criteria and as the result the diagnosis is heterogeneous. One of the major sources of heterogeneity is whether or not evidence for amyloid is present when measured by the positron emission tomography (PET). Recent biomarker studies (Salloway and Sperling, 2015 ) estimate that $36 \%$ of ApoE $\varepsilon 4$ non-carriers and $6 \%$ of ApoE $\varepsilon 4$ carriers diagnosed with AD do not have evidence for amyloid as measured by PET.

It is possible that the AD-symptoms with and without amyloid evidence have the same genetic basis and hence the clinical diagnosis is a surrogate of the disease states. It is possible, however, that the AD-symptoms underlined by the amyloid evidence and the ADsymptoms with no amyloid evidence have distinct genetic bases. We are interested to examine the role of the genetic variants serving the innate immune system in susceptibility to $\mathrm{AD}$, i.e. the $\mathrm{AD}$ symptoms underlined by the amyloid deposition. We, hence, define the disease states to be: 1) disease state of interest: AD symptoms with amyloid evidence; 2) nuisance disease state: AD symptoms with no amyloid evidence; and 3) healthy control: no $\mathrm{AD}$ symptoms and we assume no amyloid evidence. We derive the theoretical approximation that provides a simple and general relationship between the parameter estimates obtained in a model with the clinically diagnosed disease status as an outcome variable and the estimates in a model with the disease states as an outcome. The derivation is based on Kullback-Leibler divergence (Kullback, 1959).

Our paper is organized as follows. First, in the Material and Methods section we present the setting, notation, and the proposed approximation for various models. Next, in the Simulation Experiments section we describe the empirical studies that are conducted to compare the resulting performance of the derived approximation that we derived relative to the empirical estimates. We then compare the estimates in a practical setting of an Alzheimer's disease study that aims to investigate the genetic basis of innate immune system in the relationship to the AD symptoms underlined by amyloid pathology. We conclude our paper with brief Discussion.

\section{MATERIALS AND METHODS}

We define $G$ to be the genotype of single nucleotide polymorphisms (SNPs) measured at multiple locations. Let $X$ and $Z$ be the environmental variables that might interact. We assume that the genotype is independent of the environment and follows Hardy-Weinberg equilibrium model $Q(g ; \theta)$, where $\theta$ is the frequency of the minor allele. 
We define $D^{C L}$ be the observed clinical diagnosis that is inferred based on a set of descriptive criteria that characterize symptoms. Let $D$ denote the true disease states, where $D$ $=1$ indicates the disease state of interest and $D=1 *$ is the nuisance disease state. It might not be possible to measure $D$ on the whole set of cases in a GWAS, instead $D$ is available on a subset or the frequencies of $D$ within the clinically defined set of cases are reliably estimated in an external reliability study. We define the clinical-pathological diagnosis relationship using $\tau(X)=\operatorname{pr}\left(D=1 \mid D^{C L}=1, X\right)$, which is the frequency of the disease state of interest within the clinically diagnosed set and the frequency varies by $X$. In the context of AD study, $\operatorname{pr}\left(D=1 * \mid D^{C L}=1, X\right)=1-\tau(X)$, $\operatorname{pr}\left(D=0 \mid D^{C L}=1, X\right)=0, \quad \operatorname{pr}\left(D=1^{*} \mid D^{C L}=0, X\right)=\operatorname{pr}\left(D=1 \mid D^{C L}=0, X\right)=0$ and $\operatorname{pr}\left(D=1^{*} \mid D^{C L}=0, X\right)=\operatorname{pr}\left(D=0 \mid D^{C L}=0, X\right)=1$. We define the probabilities of the clinical diagnosis in the population to be ${ }_{d} C L=p r\left(D^{C L}=d^{c l}\right)$. Similarly, we let the frequencies of the true pathologic state in the population to be $\pi_{d}=\operatorname{pr}(D=d)$.

We described the relationship between the clinical diagnosis and the disease states in the context of Alzheimer's disease. The formulation can be easily extended to other settings. For example, recent biomarker studies described the disease states of breast cancer by HER/PR/ER status. The status might only be measured on subset of the cases. According to the Surveillance, Epidemiology, and End Results (SEER) database, the frequencies of the disease states by HER/PR/ER status vary by age and race/ethnicity. Hence in the context of breast cancer the relationship $\operatorname{pr}\left(D=\mathrm{d} \mid D^{C L}=1, X\right)$ is a function of $X=\{$ age, race/ethnicity $\}$. For some of the diseases, the disease states can be inferred based on the mutation pattern. The Cancer Genome Atlas, for example, archives information about mutation pattern on cancer patients. Disease states of a particular cancer type might be inferred based on mutation patterns. In this setting, the frequencies of the disease states within the clinically diagnosed set of clinically diagnosed cases are a function of the genetics, e.g. $\operatorname{pr}\left(D=\mathrm{d} \mid D^{C L}=1, G\right)$.

For clarity of presentation we assume that genotype is binary to indicate presence of a minor allele and follows Hardy-Weinberg equilibrium model, environmental variables $X$ and $Z$ are Bernoulli with frequencies $\eta_{X}$ and $\eta_{Z}$, respectively. In the Appendix we discuss how to extend the approximation to the categorical and continuous variables.

We will collectively refer to the coefficients in the disease risk model for the disease state of interest as $B$, in to the nuisance disease state as $A$ and in the model with the clinical diagnosis as an outcome as $\Gamma$.

\section{Model 1.}

$\beta_{G}$ : We first consider a setting when only the genetic variable $G$ is in the risk model, i.e. the true disease risk model is 


$$
\begin{aligned}
& \log \left\{\frac{p r_{B, A}(D=1 \mid G)}{p r_{B, A}(D=0 \mid G)}\right\}=\beta_{0}+\beta_{G} \times G ; \\
& \log \left\{\frac{p r_{B, A}\left(D=1^{*} \mid G\right)}{p r_{B, A}(D=0 \mid G)}\right\}=\alpha_{0}+\alpha_{G} \times G ;
\end{aligned}
$$

while the model used is the usual logistic regression model with the clinical diagnosis as an outcome variable, i.e.

$$
\log \left\{\frac{p r_{\Gamma}\left(D^{C L}=1 \mid G\right)}{p r_{\Gamma}\left(D^{C L}=0 \mid G\right)}\right\}=\gamma_{0}+\gamma_{G} \times G
$$

Derivations provided in Appendix A1 show that

$$
\begin{gathered}
\gamma_{0} \approx \log \left\{\exp \left(\beta_{0}\right)+\exp \left(\alpha_{0}\right)\right\} ; \quad \text { (4a) } \\
\gamma_{G} \approx \log \left\{\exp \left(\beta_{0}+\beta_{G}\right)+\exp \left(\alpha_{0}+\alpha_{G}\right)\right\}-\log \left\{\exp \left(\beta_{0}\right)+\exp \left(\alpha_{0}\right)\right\} \\
\approx \log \left\{\exp \left(\beta_{0}\right)+\exp \left(\alpha_{0}+\alpha_{G}\right)\right\}-\log \left\{\exp \left(\beta_{0}\right)+\exp \left(\alpha_{0}\right)\right\}+\frac{\exp \left(\beta_{0}\right)}{\exp \left(\beta_{0}\right)+\exp \left(\alpha_{0}+\alpha_{G}\right)} \times \beta_{G} .
\end{gathered}
$$

From (4a) and (4b), we derive that

$$
\begin{gathered}
\beta_{0} \approx \log \left\{\exp \left(\gamma_{0}\right)-\exp \left(\alpha_{0}\right)\right\} ; \quad(4 \mathrm{c}) \\
\beta_{G} \approx \log \left\{\exp \left(\gamma_{0}+\gamma_{G}\right)-\exp \left(\alpha_{0}+\alpha_{G}\right)\right\}-\log \left\{\exp \left(\gamma_{0}\right)-\exp \left(\alpha_{0}\right)\right\} .
\end{gathered}
$$


Appendix A3 describes how to obtain $\beta_{0}, \beta_{G}, a_{0}, a_{G}$ assuming estimates of $\gamma_{0}, \gamma_{G}$ are available from the usual logistic regression and reliable estimates of $\tau=\tau(1) \times \operatorname{pr}(X=1)+\tau(0) \times \operatorname{pr}(X=0)$ and $\pi_{1}$ are available in the literature.

\section{Model 2.}

$\beta_{G}$ and $\beta_{X}$ : We next consider a setting when the genetic variable $G$ and an environmental variable $X$ are in the risk model, i.e. the true disease risk model is

$$
\begin{aligned}
& \log \left\{\frac{p r_{B, A}(D=1 \mid G, X)}{p r_{B, A}(D=0 \mid G, X)}\right\}=\beta_{0}+\beta_{G} \times G+\beta_{X} \times X ; \\
& \log \left\{\frac{p r_{B, A}\left(D=1^{*} \mid G, X\right)}{p r_{B, A}(D=0 \mid G, X)}\right\}=\alpha_{0}+\alpha_{G} \times G+\alpha_{X} \times X ;
\end{aligned}
$$

while the model used is

$$
\log \left\{\frac{p r_{\Gamma}\left(D^{C L}=1 \mid G, X\right)}{p r_{\Gamma}\left(D^{C L}=0 \mid G, X\right)}\right\}=\gamma_{0}+\gamma_{G} \times G+\gamma_{X} \times X .
$$

Derivations provided in Appendix A2 show that

$$
\begin{gathered}
\gamma_{0} \approx \log \left\{\exp \left(\beta_{0}\right)+\exp \left(\alpha_{0}\right)\right\} ; \quad \text { (8a) } \\
\gamma_{G} \approx 0.5 \times \sum_{x}\left[\log \left\{\exp \left(\beta_{0}+\beta_{G}+\beta_{X} \times x\right)+\exp \left(\alpha_{0}+\alpha_{G}+\alpha_{X} \times x\right)\right\}-\log \left\{\operatorname { e x p } \left(\beta_{0}+\beta_{X} \times\right.\right.\right. \\
\left.\approx 0.5 \times \sum_{x}\left[\exp \left(\alpha_{0}+\alpha_{X} \times x\right)\right\}\right] \\
\left.\left.\exp \left(\alpha_{0}+\alpha_{X} \times x\right)\right\}\right]+0.5 \times \sum_{x} \frac{\left.\exp \left(\beta_{0}+\beta_{X} \times x\right)+\exp \left(\alpha_{0}+\alpha_{G}+\alpha_{X} \times x\right)\right\}-\log \left\{\exp \left(\beta_{0}+\beta_{X} \times x\right)+\right.}{\exp \left(\beta_{0}+\beta_{X} \times x\right)+\exp \left(\alpha_{0}+\alpha_{G}+\alpha_{X} \times x\right)} \times \beta_{G} ;
\end{gathered}
$$




$$
\begin{array}{r}
\gamma_{X} \approx 0.5 \times \sum_{g}\left[\log \left\{\exp \left(\beta_{0}+\beta_{X}+\beta_{G} \times g\right)+\exp \left(\alpha_{0}+\alpha_{X}+\alpha_{G} \times g\right)\right\}-\log \left\{\operatorname { e x p } \left(\beta_{0}+\beta_{G} \times\right.\right.\right. \\
\left.\left.g)+\exp \left(\alpha_{0}+\alpha_{G} \times g\right)\right\}\right] \\
\approx 0.5 \times \sum_{g}\left[\log \left\{\exp \left(\beta_{0}+\beta_{G} \times g\right)+\exp \left(\alpha_{0}+\alpha_{X}+\alpha_{G} \times g\right)\right\}-\log \left\{\exp \left(\beta_{0}+\beta_{G} \times g\right)+\right.\right. \\
\left.\left.\exp \left(\alpha_{0}+\alpha_{G} \times g\right)\right\}\right]+0.5 \times \sum_{x} \frac{\exp \left(\beta_{0}+\beta_{G} \times g\right)}{\exp \left(\beta_{0}+\beta_{G} \times g\right)+\exp \left(\alpha_{0}+\alpha_{X}+\alpha_{G} \times g\right)} \times \beta_{X} .
\end{array}
$$

\section{Model 3.}

$\beta_{G}, \beta_{X}, \beta_{Z}$, and $\beta_{X \times Z .:}$ A model with interaction between the environmental variables $X$ and $Z$ is discussed in Appendix.

\section{Model 4.}

$\beta_{G_{1}}, \beta_{G_{2}}$ and $\beta_{G_{1} \times G_{2}}$ : A model with gene-gene interactions is discussed in Appendix.

\section{Remarks:}

1. Model 1, equation (4b). If $\beta_{G}=\alpha_{G}=0$, then $\gamma_{G}=0$.

2. Model 2, equation (8b). If $\beta_{G}=\alpha_{G}=0$, then $\gamma_{G}=0$.

3. Model 2, equation (8c). If $\beta_{X}=\alpha_{X}=0$, then $\gamma_{X}=0$.

4. Remarks 1-3 describe when the usual logistic regression models with the clinical diagnosis as an outcome variable correctly estimate the null effect.

5. The equations that we derived apply to several possible likelihood functions. For example, parameter estimates in Model 3 can be estimated based on the usual logistic regression model, i.e. the probability of the form $\operatorname{pr}_{\Gamma}\left(D^{C L} \mid G, X, Z\right)$ or in a pseudolikelihood (Spinka et al, 2005; Lobach et al, 2018) $\operatorname{pr}_{\Gamma}\left(D^{C L}, G \mid X, Z, \delta=1\right)$, where, $\delta=1$ is an imaginary indicator of being selected into the study. All the derivations apply to both models.

\section{SIMULATION STUDIES}

\section{False positive rate}

We first perform a series of simulation experiments to examine a false positive rate in the estimates of $\beta_{G}$ when the data are simulated from model (1)-(2), but the parameter estimates are obtained from model (3). We define the false positive rate to be the fraction of $\mathrm{p}$ - 
values $₫ 0.05$ across 10,000 simulated datasets in the usual logistic regression analyses as an outcome variable, i.e. (3), when in fact $\beta_{G}=0$. We simulate the data using model (1) with coefficients $\beta_{0}=0.5, \quad \beta_{G}=0, \quad \alpha_{G}=\log (1)=0, \log (1.5)=0.41, \log (2)=0.69$. We next estimate parameters using model (3). Table 1 presents false positive rates in datasets with $n_{0}=n_{1}=3,000 ; 10,000$. When the genetic effect is not associated with the clinical diagnosis, the false positive rate is nominal, i.e. is nearly 0.05 . When $a_{G}$ increases, the false positive rate gets inflated, e.g. when $\alpha_{G}=\log (1.5)=0.41$, the false positive rate is 0.72 . Increase in sample size did not result in decrease of the false positive rate.

\section{Approximation vs. empirical estimates}

We next perform a series of simulation experiments to assess the magnitude of bias and the approximation to the relationships that we've derived. First, we estimate the bias empirically as the average across 500 simulated datasets where the data are simulated using the true model (1)-(2), (5)-(6), (A3)-(A4) based on coefficients $B$ and $A$, but estimate the parameters $\Gamma$ in the usual logistic regression model (3), (7) and (A5). We then compare these averages to the approximations that we've derived.

We simulate genotype $(G)$, age $(A)$, sex $(S)$, ApoE $\epsilon 4$ status to be Bernoulli with frequencies $\theta_{G}, \theta_{A}, \theta_{S}, \theta_{\epsilon 4}$. In the context of previous notations, $X$ is the ApoE $\epsilon 4$ status and $Z$ is a set consisting of $G, A, S$. We then simulated the clinical diagnosis status $D^{C L}$ according to the models (3), (7) and (A5) and the true disease states $D$ according to model (1)-(2), (5)-(6), (A3)-(A4). In all simulations we let $\theta_{G}=0.10, \theta_{A}=0.50, \theta_{S}=0.52, \theta_{\epsilon 4}=0.07$.

\section{Model 1}

We first consider models with one genetic variable. We fist simulate the data using model (1)-(2) and estimate parameters in the logistic model (3). We set $\beta_{0}=0.5$,

$\beta_{G}=\log (1)=0, \log (1.5)=0.41, \log (2)=0.69, \log (2.5)=0.92, \log (3)=1.1$,

$\alpha_{G}=\log (1)=0, \log (1.5)=0.41,=0.69$ and simulate datasets with 3,000 cases and 3,000 controls. Table 2 presents empirical estimates of $\beta_{G}$ and the approximation (4b). Across all values of $\beta_{G}$ and $a_{G}$, the approximation (4b) is accurate relative to the empirical estimate.

\section{Model 2}

We first consider models with one genetic variable and one environmental variable. We next generate data using models (5)-(6) but estimate parameters using model (7). We let $\beta_{0}=\alpha_{0}=0.5$, $\beta_{G}=\log (1)=0, \log (1.5)=0.41, \log (2)=0.69, \log (2.5)=0.92, \log (3)=1.1, \quad \beta_{\epsilon 4}=\alpha_{\epsilon 4}=\log (8)$, $\alpha_{G}=\log (1)=0, \log (2)=0.41, \log (3)=0.69, \log (4)=1.1$

and generate datasets with 3,000 cases and 3,000 controls. Approximations and the empirical estimates for $\gamma_{G}$ shown in Table 3 demonstrate that the approximation ( $8 \mathrm{~b}$ ) is accurate relative to the empirical estimates. The empirical estimate of $\gamma_{\epsilon 4}{ }^{\epsilon}$ is 2.09 , while the approximation is 2.08 . 


\section{Model 3}

We next consider a model with one genetic variable and two environmental variables that interact. We next simulate data using models (A3)-(A4) and estimate parameters using model (A5), with the approximation derived in $(\mathrm{A} 6 \mathrm{a}-\mathrm{c})$.

Setting 1.-We first consider a setting when the genotype is not associated with the nuisance disease ( $a_{G}=0$ ) and when $\epsilon 4$ and $A \times € 4$ are not associated with the nuisance disease status $\left(\alpha_{\epsilon 4}=0, \quad \alpha_{G}=0, \alpha_{A \times \epsilon 4}=0\right)$. We simulate the clinical diagnosis and disease states with coefficients

$\beta_{0}=-1, \beta_{S}=\log (0.92)=-0.08, \beta_{\epsilon 4}=\log (8)=2.1, \beta_{A}=\log (2)=0.69$,

$$
\beta_{G}=\log (1), \log (1.5), \log (2), \log (2.5), \log (3), \beta_{A \times \epsilon 4}=\log (1), \log (2), \log (3), \log (3),
$$

$$
\alpha_{0}=-1, \alpha_{S}=\log (0.92), \alpha_{\epsilon 4}=0, \alpha_{A}=\log (2), \alpha_{G}=0, \alpha_{A \times \in 4}=0 .
$$

Figure 1 presents biases in estimates of $\beta_{G}$ (panel A), $\beta_{A}$ (panel B), $\beta_{S}$ (panel C), $\beta_{\epsilon 4}$ (panel D) and $\beta_{A \times \in 4}$ (panel E) in studies with 3,000 cases and 3,000 controls; values of $\beta_{A \times \in 4}$ are shown along the $\mathrm{x}$-axis and values of $\beta_{G}$ are indicated by color. Figure 1 panels $\mathbf{A}$ and $\mathbf{D}$ show that bias in the estimates of $\beta_{G}$ and $\beta_{\epsilon 4}$ can be substantial with largest bias of -0.06 ; panel $\mathbf{E}$ shows that bias in $\beta_{A \times \in 4}$ is notable in this case ranging from 0.01 to -0.06 ; estimates of $\beta_{A}$ and $\beta_{S}$ are nearly unbiased consistent with the theoretical observations that the null effect in some settings can be estimated with no bias even in a misspecified model. We note that magnitude of bias in $\widehat{\beta_{G}}$ and $\widehat{\beta_{A \times \varepsilon 4}}$ increases as the true value of the coefficient increases.

Shown on Figure 2 are the empirical bias (Emp) and the approximation (AX) of bias in $\beta_{G}$ indicated by color with values of $\beta_{A \times \in 4}$ along the $\mathrm{x}$-axis and values of $\beta_{G}$ along the panels. The difference between the Emp and AX starts at $\approx 0.6$ when $\beta_{G}=0.41$ and increases to $\approx$ 1.2 when $\beta_{G}=1.1$. Bias of $\widehat{\beta_{S}}$ and $\widehat{\beta_{A}}$ is approximated to be $<0.0001$. Shown on Figure 3 are Emp and $\mathrm{AX}$ of estimates of $\beta_{\epsilon 4}$, and Figure 4 is presenting estimates of $\beta_{A \times \in 4}$.

Setting 2.-We next simulate datasets with 30,000 cases and 30,000 controls in the Setting 1. Supplementary Figure 1 shows that biases in the estimates noted in Setting 1 persists for larger sample sizes.

Setting 3.-We next consider a setting when the genotype and environment are associated with the nuisance disease state $\left(a_{G}=\log (1.5), \alpha_{\epsilon 4}=\log (2)\right)$, but no interaction $\alpha_{A \times \epsilon 4}=0$. We next change the parameters for the nuisance state to be $\alpha_{0}=-1, \alpha_{S}=\log (0.92), \alpha_{\epsilon 4}=\log (2), \alpha_{A}=\log (2), \alpha_{G}=\log (1.5), \alpha_{A \times \epsilon 4}=0$ and all other parameters as in Setting 1. Shown on Supplementary Figure 2 are biases in the estimates of 
the parameters of interest that reach -1.4 for $\widehat{\beta_{G}}$, are near -0.5 for $\widehat{\beta_{\epsilon 4}}$, and are up to -0.15 for $\overline{\beta_{A \times \varepsilon 4}}$.

Setting 4.-We next consider a Setting 1 but with more common disease of interest, i.e. $\beta_{0}$ $=1.5$. Supplementary Figure 3 is showing empirical bias in all estimates, which can still be substantially biased.

Setting 5.-We next consider a setting where the genetic variable is associated with the nuisance disease state $\left(\alpha_{G}=\log (2)\right)$ and there is a significant $A \times \epsilon 4$ interaction

$\left(\alpha_{A \times \in 4}=\log (2)\right)$. We next change the parameters for the nuisance state to be

$\alpha_{0}=0.5, \alpha_{S}=\log (0.80), \alpha_{\epsilon 4}=\log (4), \alpha_{A}=\log (3), \alpha_{G}=\log (2), \alpha_{A \times \epsilon 4}=\log (2)$. Figure 5

presents biases in the estimates and Supplementary Figures 4-5 show the empirical estimates and the approximations.

Supplementary Figure 7: Frequency of the disease state of interest $(D=1)$ and the nuisance disease $\left(D=1^{*}\right)$ when $\beta_{0}=1.5, \beta_{S}=\log (0.80), \beta_{\epsilon 4}=\log (8), \beta_{A}=\log (3)$,

$$
\begin{aligned}
& \beta_{G}=\log (1), \log (1.5), \log (2), \log (2.5), \log (3), \beta_{G \times \in 4}=\log (1), \log (2), \log (3), \log (3), \\
& \alpha_{0}=0.5, \alpha_{S}=\log (0.80), \alpha_{\epsilon 4}=\log (4), \alpha_{A}=\log (3), \alpha_{G}=\log (2), \alpha_{A \times \epsilon 4}=\log (2),
\end{aligned}
$$

$\theta_{G}=0.10, \theta_{A}=0.50, \theta_{S}=0.52, \theta_{\epsilon 4}=0.07$. Shown along the $\mathrm{x}$-axis are values of $\beta_{G}$ and indicated by color are values of $\beta_{A \times \epsilon 4}$. We note that these frequencies are similar to those in context of Alzheimer's disease.

\section{ROLE OF THE GENETIC VARIANTS SERVING INNATE IMMUNE SYSTEM IN SUSCEPTIBILITY TO ALZHEIMER'S DISEASE}

We apply the usual logistic analyses with the clinical diagnosis as an outcome variable to a dataset collected as part of the Alzheimer's Disease Genetics Consortium. We next apply the approximations (7)-(10) and (11)-(14) to see how the genetic estimates change when presence of the nuisance disease state is recognized.

We mapped Illumina Human 660K markers onto human chromosomes using NCBI dbSNP database (https://www.ncbi.nlm.nih.gov/projects/SNP/). Chromosomal location, proximal gene or genes and gene structure location (e.g. intron, exon, intergenic, UTR) has been recorded for all SNPs. From these data we inferred 165 SNPs to reside in genes serving innate immune system. 


\section{The dataset consists of 727 controls and 2,797 cases diagnosed with AD.}

We are interested to examine a relationship between the pathologic disease state of $\mathrm{AD}$ characterized by presence of amyloid deposition and each of the 165 SNPs serving the innate immune system. We include ApoE $\epsilon 4$ status, age, and sex in the model with an interaction between ApoE and age. The genetic variant is modeled as a Bernoulli variable as an indicator of presence or absence of a minor allele. Age is Bernoulli as well that corresponds to a median split in the dataset.

Table 4 presents estimates of effects of the SNPs obtained using the usual logistic regression model with the clinical diagnosis as an outcome variable in a univariable model (3) and with adjustment for SNP + ApoE $\varepsilon 4+$ Age + Sex (7); and the corresponding models (1-2) and (5-6) that recognize presence of the nuisance disease state. In the univariable setting the empirical bias is estimated as the difference between the main effect estimates obtained in model (3) and model (1-2), and the approximation to the bias is estimated as derived in (4b). In the multivariable setting, the empirical bias is the difference between main effect estimates obtained in model (7) and (5-6), and the approximation is as derived in (8b).

First shown in Table 4 are 16 estimates with p-value $<0.05$ after the Benjamini-Hochberg multiple testing adjustment in a univariable model (3) and then added are 13 SNPs with pvalue $<0.05$ in a univariable model (1-2). Across all these SNPs, the approximation was accurate relative to the empirical bias.

We describe the findings further in Web-based Supplementary file. In section "Alzheimer's disease study" we describe which variants have been previously reported in the literature. We also discuss the 11 variants from Table 4 relevant to amyloid protein and 5 variants from Table 4 relevant to tau protein.

\section{DISCUSSION}

We've examined a situation when multiple disease states share observed symptoms and hence the clinical diagnosis. Both theoretically and in extensive simulation studies we observed that the magnitude of bias can be substantial in the situations when the frequency of the nuisance disease state within the clinically diagnosed set varies by the key variables. We derived a simple and general approximation to the relationship between the genetic effect estimates that use the clinical diagnosis as an outcome variable and the estimates that recognize the presence of the nuisance disease state.

While the effect of misclassification of the disease status has been examined extensively in statistical literature (Carroll et al, 2006), we extend the literature by deriving a simple and general approximation to the bias in a multivariable setting. The approximation provides a simple formula to assess how elastic the estimates of interest are to the values of parameters in the nuisance risk model. The regression coefficients or plausible ranges for the coefficients of the nuisance disease state are often available in the literature.

In the derivations we assume that the link functions and disease risk models for the clinical diagnosis and the disease risk are the same, for example, logit and additive with the same 
variables. The derivations are readily generalizable to the settings when the link function is the same, but the disease risk models are different. The generalization to the different link functions, however, is out of the scope of the current paper and requires additional derivations and is subject to future studies.

Simulation studies that we conducted showed that when the presence of the nuisance disease is ignored, the genetic effect estimates can be biased in either direction. These biases can be substantial in magnitude leading to false positive and false negative results. The magnitude of bias in the genetic effect estimates is a function of the frequencies of the disease state of interest and the nuisance disease state in the population (through the intercept), coefficients of the genetics in the nuisance disease state model, and coefficients of the environment both in the disease state of interest and the nuisance disease state. The interplay between all of these parameters in the form of the approximation that we've derived determines the magnitude and directionality of the bias. Importantly, the magnitude of bias persists in large sample sizes.

The approximation relies on the external information published in the literature. In situations when the external estimates are deemed not reliable, we advocate for sensitivity analyses by varying the external estimates and assessing the change in the genetic estimates of interest.

The form of the approximation that we've derived is simple and such analyses can be easily performed. For example, the parameters in the nuisance model can be set on a plausible interval and the degree of change in the estimates of interest can then be assessed. Often in practice the actual values of the coefficients are not of interest per se. Instead, what is of interest is the relative order of the genetic variants according to their magnitude of effect. Hence it might be useful to assess how the relative order of the estimates changes when the nuisance parameters are varied on a plausible range.

While our study is motivated by the setting of Alzheimer's disease, the results are readily applicable for other complex diseases. For example, Manchia el al (2013) examined the effect of heterogeneity, i.e. presence of non-cases, in the context of diabetes and showed that ignoring the heterogeneity leads to reduced statistical power to detect an association and also reduced the estimated risks attributable to susceptibility alleles.

The approximation that we've derived is widely applicable in other areas of research where the diagnosis is heterogeneous. For example, when disease states correspond to subtypes of a complex disease. We also see the application to the analyses of Electronic Health Records, where the disease status might be subject to exposure-dependent differential misclassification (Chen et al, 2017).

\section{Supplementary Material}

Refer to Web version on PubMed Central for supplementary material.

\section{ACKNOWLEDGEMENTS}

Dr. Lobach is supported by 5R21AG043710-02. 
Genotyping is performed by Alzheimer's Disease Genetics Consortium (ADGC), U01 AG032984, RC2 AG036528. Phenotypic collection is coordinated by the National Alzheimer's Coordinating Center (NACC), U01 AG016976

Samples from the National Cell Repository for Alzheimer's Disease (NCRAD), which receives government support under a cooperative agreement grant (U24 AG21886) awarded by the National Institute on Aging (NIA), were used in this study. We thank contributors who collected samples used in this study, as well as patients and their families, whose help and participation made this work possible;

Data for this study were prepared, archived, and distributed by the National Institute on Aging Alzheimer's Disease Data Storage Site (NIAGADS) at the University of Pennsylvania (U24-AG041689-01)

We thank Ivan Belousov for help with the computations.

\section{APPENDIX}

\section{A1. Approximation using Kullback-Leibler divergence}

We show schematics of the derivations based on Model 3, the other models can be derived accordingly. We denote the model the true model (9)-(10) based on probability $p r_{\Gamma}\left(D^{C L} \mid G, X, Z\right)$ or $p r_{\Gamma}\left(D^{C L}, G \mid X, Z, \delta=1\right)$ as $Q_{B, A}\left(D^{C L}, G, X, Z\right)=p r_{B, A}\left(D^{C L}, G \mid X, Z, \delta=1\right)$.

Similarly, we denote model (3) with (4) as $Q_{\Gamma}\left(D^{C L}, G, X, Z\right)=p r_{\Gamma}\left(D^{C L} \mid G, X, Z\right)$.

Kullback (1959) showed that parameters $\Gamma$ converge to values that minimize KullbackLeibler divergence criteria between the two models, specifically

$$
\gamma=\operatorname{argmin}\left\{E_{G, X, Z}\left(\left.E_{D}^{C L}\right|_{G, X, Z}\left[\log \left\{\frac{Q_{B, A}\left(D^{C L}, G, X, Z\right)}{Q_{\Gamma}\left(D^{C L}, G, X, Z\right)}\right\}\right)\right\}\right) .
$$

Considerable algebraic derivations arrive to the following system of equations to be solved for parameters $\Gamma$

$$
\begin{aligned}
E_{G, X, Z}\left[\frac{\left[p_{B, A}(D=1 \mid G, X, Z)+p r_{B, A}\left(D=1^{*} \mid G, X, Z\right)\right] \times \operatorname{pr}(G)}{p r_{\Gamma}\left(D^{C L}=1 \mid G, X, Z\right)}\right. \\
\quad \times \frac{\partial}{\partial \Gamma} \frac{\exp \left(\gamma_{0}+\gamma_{X} \times X+\gamma_{G} \times G+\gamma_{Z} \times Z+\gamma_{X \times Z} \times X \times Z\right)}{1+\exp \left(\gamma_{0}+\gamma_{X} \times X+\gamma_{G} \times G+\gamma_{Z} \times Z+\gamma_{X} \times Z^{\times X \times Z)}\right.} \\
\quad+\frac{p r_{B, A}(D=0 \mid G, X, Z) \times p r(G)}{p r_{\Gamma}\left(^{C L}=0 \mid G, X, Z\right)} \\
\left.\quad \times \frac{\partial}{\partial \Gamma} \frac{1}{1+\exp \left(\gamma_{0}+\gamma_{X} \times X+\gamma_{G} \times G+\gamma_{Z} \times Z+\gamma_{X} \times Z^{\times X \times Z)}\right)}\right]=0
\end{aligned}
$$

(A1)

Define $M(X, G, Z ; \Gamma)=\operatorname{pr}(G) \times \frac{\exp \left(\gamma_{0}+\gamma_{X} \times X+\gamma_{G} \times G+\gamma_{Z} \times Z+\gamma_{X \times Z} \times X \times Z\right)}{1+\exp \left(\gamma_{0}+\gamma_{X} \times X+\gamma_{G} \times G+\gamma_{Z} \times Z+\gamma_{X \times Z} \times X \times Z\right)}$ Then (A1) becomes 


$$
\begin{aligned}
& E_{G, X, Z}\left[X \times M(X, G, Z ; \Gamma)\left\{\frac{p r_{B, A}(D=1 \mid G, X, Z)+p r_{B, A}\left(D=1^{*} \mid G, X, Z\right)}{p r_{\Gamma}\left(D^{C L}=1 \mid G, X, Z\right)}\right.\right.
\end{aligned}
$$

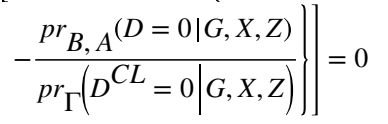

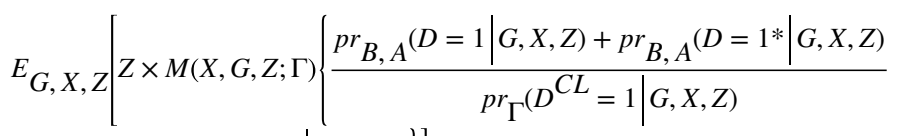

$$
\begin{aligned}
& \left.-\frac{p r_{B, A}(D=0 \mid G, X, Z)}{p r_{\Gamma}\left(D^{C L}=0 \mid G, X, Z\right)}\right\} \mid=0 \\
& E_{G, X, Z}\left[G \times M(X, G, Z ; \Gamma)\left\{\frac{p r_{B, A}(D=1 \mid G, X, Z)+p r_{B, A}(D=1 * \mid G, X, Z)}{\left.p r_{\Gamma^{(D}} D^{C L}=1 \mid G, X, Z\right)}\right.\right. \\
& \left.-\frac{p r_{B, A}(D=0 \mid G, X, Z)}{p r_{\Gamma}\left(D^{C L}=0 \mid G, X, Z\right)}\right\} \mid=0 \\
& E_{G, X, Z}\left[X \times Z \times M(X, G, Z ; \Gamma)\left\{\frac{p r_{B, A}(D=1 \mid G, X, Z)+p r_{B, A}\left(D=1^{*} \mid G, X, Z\right)}{p r_{\Gamma}\left(D^{C L}=1 \mid G, X, Z\right)}\right.\right. \\
& \left.\left.-\frac{p r_{B, A}(D=0 \mid G, X, Z)}{p r_{\Gamma}\left(D^{C L}=0 \mid G, X, Z\right)}\right\}\right]=0
\end{aligned}
$$

Values of $\Gamma$ such that

$$
\frac{\left.p r_{B, A^{(D=1}} \mid G, X, Z\right)+p r_{\left.B, A^{(D=1 *} \mid G, X, Z\right)}}{p r_{\Gamma}\left(D^{C L}=1 \mid G, X, Z\right)}=\frac{\left.p r_{B, A^{(D=0}} \mid G, X, Z\right)}{\operatorname{pr}_{\Gamma}{ }^{\left(D^{C L}=0 \mid G, X, Z\right)}}=1
$$

for all $G, X, Z$ solve the system of equations (A1).

By definition,

$$
\begin{gathered}
\gamma_{G}=0.25 \times \sum_{x, z}\left[\operatorname{logit}\left\{p r_{\Gamma}\left(D^{C L}=1 \mid G=1, X=x, Z=z\right)\right\}-\operatorname{logit}\left\{\operatorname { p r } _ { \Gamma } \left(D^{C L}=1 \mid G=0, X\right.\right.\right. \\
=x, Z=z)\}] .
\end{gathered}
$$

With Taylor series expansion around $\beta_{G}=0$ we arrive at (12a). Derivation for the other parameters is similar. If $X$ is continuous, then e.g., 


$$
\begin{aligned}
& \gamma_{X}=0.5 \times \sum_{g}\left[\operatorname{logit}\left\{p r_{\Gamma}\left(D^{C L}=1 \mid G=g, X=x+1, Z=0\right)\right\}-\operatorname{logit}\left\{\operatorname { p r } _ { \Gamma } \left(D^{C L}=1 \mid G=g, X=\right.\right.\right. \\
& x, Z=0)\}] .
\end{aligned}
$$

\section{LITERATURE CITATIONS}

Carroll RJ, Ruppert D, Stefanski LA, Crainiceanu (2006) Measurement error in nonlinear models: a modern perspective, Second Edition, Chapman and Hall/CRC

Chen Y, Wang J, Chubak J, Hubbard RA (2018) Inflation of type I error rates due to differential misclassification in HER-derived outcomes: empirical illustration using breast cancer recurrence, Pharmacoepidemiiol Drug Saf, 2018: 1-5

Kullback S (1959) Information theory and statistics New York: John Wiley

Lobach I, Sampson J, Alexeyenko A, Lobach S, Zhang L (2018) Case-control studies of geneenvironment interactions. When a case might not be the case. PLOS One, in press

Manchia M, Cullis J, Gustavo T, Rouleau GY, Uher R, Alda M. (2013) The impact of phenotypic and genetic heterogeneity on results of genome-wide association studies of complex diseases. PLOS One 2013; 8(10): e76295. [PubMed: 24146854]

Salloway S and Sperling R (2015) Understanding conflicting neurological findings in patients clinically diagnosed as having Alzheimer Dementia. JAMA Neurology, 72 (10): 1106-8 [PubMed: 26302229]

Spinka C, Carroll RJ, Chatterjee N (2005) Analysis of case-control studies of genetic and environmental factors with missing genetic information and haplotype-phase ambiguity, Genetic Epidemiology, 29(2) 108-127 [PubMed: 16080203] 

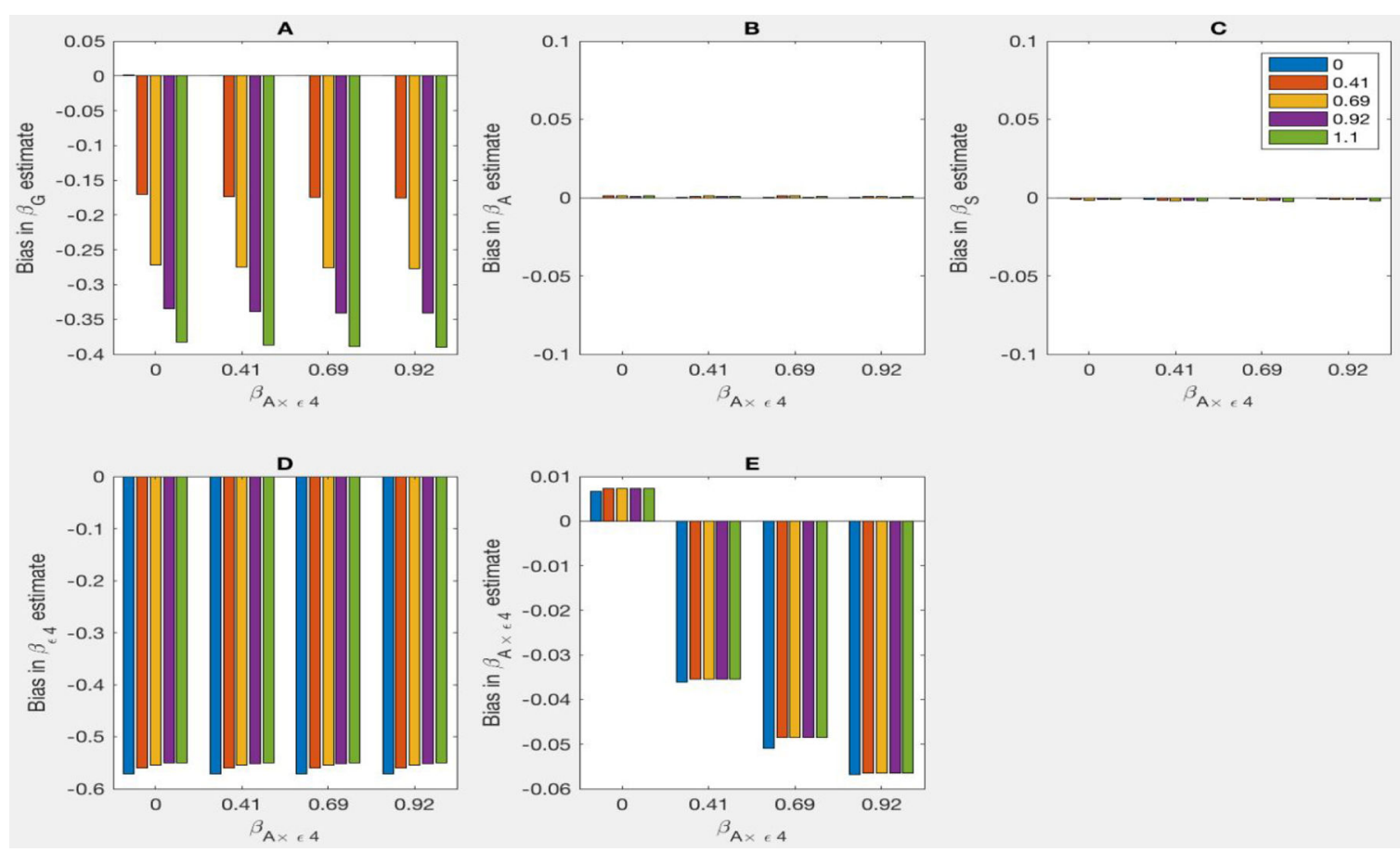

Figure 1.

Empirical biases in estimates of $\beta_{G}, \beta_{A}, \beta_{S}, \beta_{\epsilon 4}, \beta_{A \times \epsilon 4}$ across 500 datasets with 3,000 cases and 3,000 controls when the disease states are simulated according to risk model (1)-(2), but the parameters are estimated using model (3). Here the genotype, ApoE $\epsilon 4$ status and $A \times \epsilon 4$ are not associated with the nuisance disease state. Genotype, age, sex, ApoE $\epsilon 4$ status are simulated to be binary with frequencies $\theta_{G}=0.10, \theta_{A}=0.50, \theta_{S}=0.52, \theta_{\epsilon 4}=0.07$. The disease states are then simulated according to risk model (1)-(2) with $\beta_{0}=-1, \beta_{S}=\log (0.92)=-0.08, \beta_{\epsilon 4}=\log (8)=2.1, \beta_{A}=\log (2)=0.69$, $\beta_{G}=\log (1), \log (1.5), \log (2), \log (2.5), \log (3), \beta_{A \times \in 4}=\log (1)=0, \log (1.5)=0.41, \log (2)=0.69$, $\log (2.5)=0.92$,

$\alpha_{0}=-1, \alpha_{S}=\log (0.92), \alpha_{\epsilon 4}=0, \alpha_{A}=\log (2), \alpha_{G}=0, \alpha_{A \times € 4}=0$. Values of $\beta_{A \times € 4}$ are along the $\mathrm{x}$-axis and values of $\beta_{G}$ are indicated by color. 

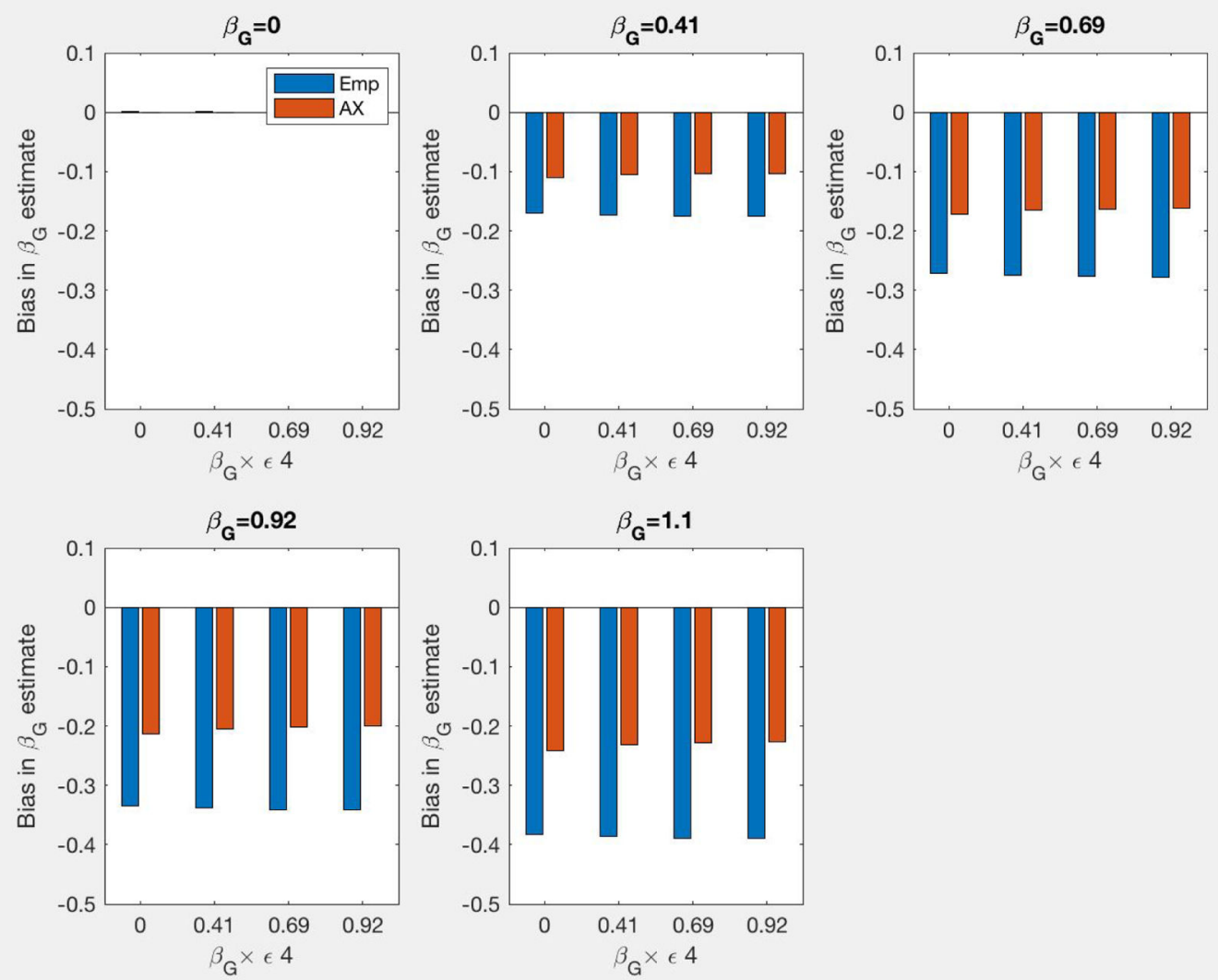

Figure 2.

Empirical bias (Emp) and bias approximated by $(\mathrm{A} 6 \mathrm{~b})(\mathrm{AX})$ in $\beta_{G}$ estimates when the data are generated according to disease states as in (A3)-(A4), but the parameters are estimated in the model (A5). Empirical estimates are the averages 500 datasets with 3,000 cases and 3,000 controls. Genotype, age, sex, ApoE $\epsilon 4$ status are simulated to be binary with frequencies $\theta_{G}=0.10, \theta_{A}=0.50, \theta_{S}=0.52, \theta_{\epsilon 4}=0.07$. The pathologically defined disease states are then simulated according to risk model (A3)-(A4) with $\beta_{0}=-1, \beta_{S}=\log (0.92)=$ $-0.08, \beta_{\epsilon 4}=\log (8)=2.1, \beta_{A}=\log (2)=0.69, \beta_{G}=\log (1)=0, \log (1.5)=0.41, \log (2)=$ $0.69, \log (2.5)=0.92, \log (3)=1.1, \beta_{A \times \epsilon 4}=\log (1)=0, \log (1.5)=0.41, \log (2.5)=0.92, a_{0}=$ $-1, a_{S}=\log (0.92), a_{\epsilon 4}=0, a_{A}=\log (2), a_{A}=0, a_{A \times \epsilon 4}=0$. Values of $\beta_{A \times \epsilon 4}$ are along the Xaxis and empirical estimates are shown in blue, approximations (A6a-c) are in red. 

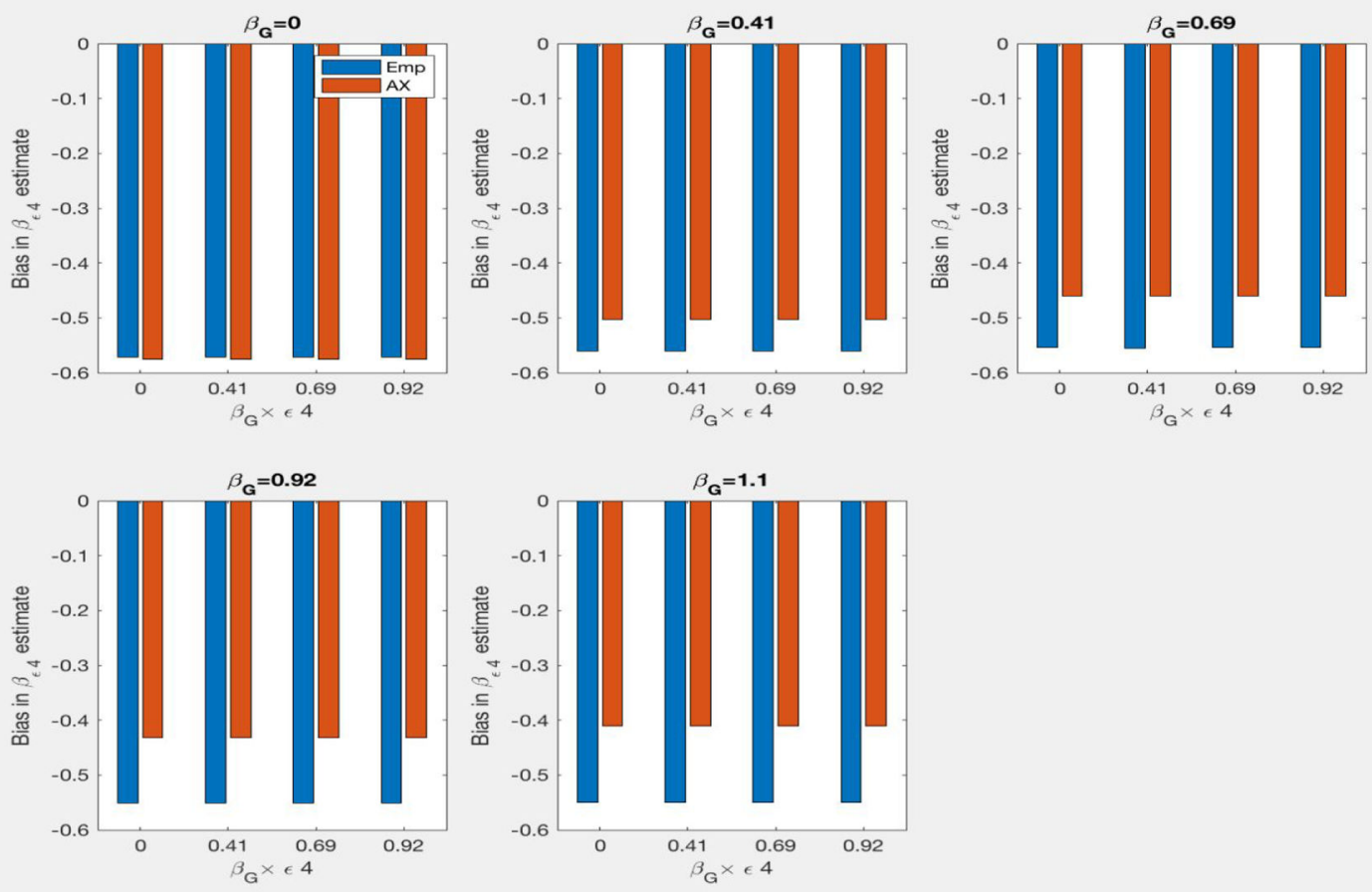

Figure 3.

Empirical bias (Emp) and bias approximated by (A6b)(AX) in $\beta_{\epsilon 4}$ estimates when the data are generated according to disease states as in (A3)-(A4), but the parameters are estimated in the model (A5). Genotype, age, sex, ApoE $\epsilon 4$ status are simulated to be binary with frequencies $\theta_{G}=0.10, \theta_{A}=0.50, \theta_{S}=0.52, \theta_{\epsilon 4}=0.07$. The pathologically defined disease states are then simulated according to risk model (A3) - (A4) $\beta_{0}=-1, \beta_{S}=\log (0.92)=$ $-0.08, \beta_{\epsilon 4}=\log (8)=2.1, \beta_{A}=\log (2)=0.69, \beta_{G}=\log (1)=0, \log (1.5)=0.41, \log (2)=$ $0.69, \log (2.5)=0.92, \log (3)=1.1, \beta_{A \times \epsilon 4}=\log (1)=0, \log (1.5)=0.41, \log (2)=0.69, \log (2.5)=$ $0.92, a_{0}=-1, a_{S}=\log (0.92), a_{\epsilon 4}=0, a_{A}=\log (2), a_{G}=0, a_{A \times \epsilon 4}=0$. Values of $\beta_{A \times \epsilon 4}$ are along the $\mathrm{x}$-axis and empirical estimates are shown in blue, approximations are in red.. 

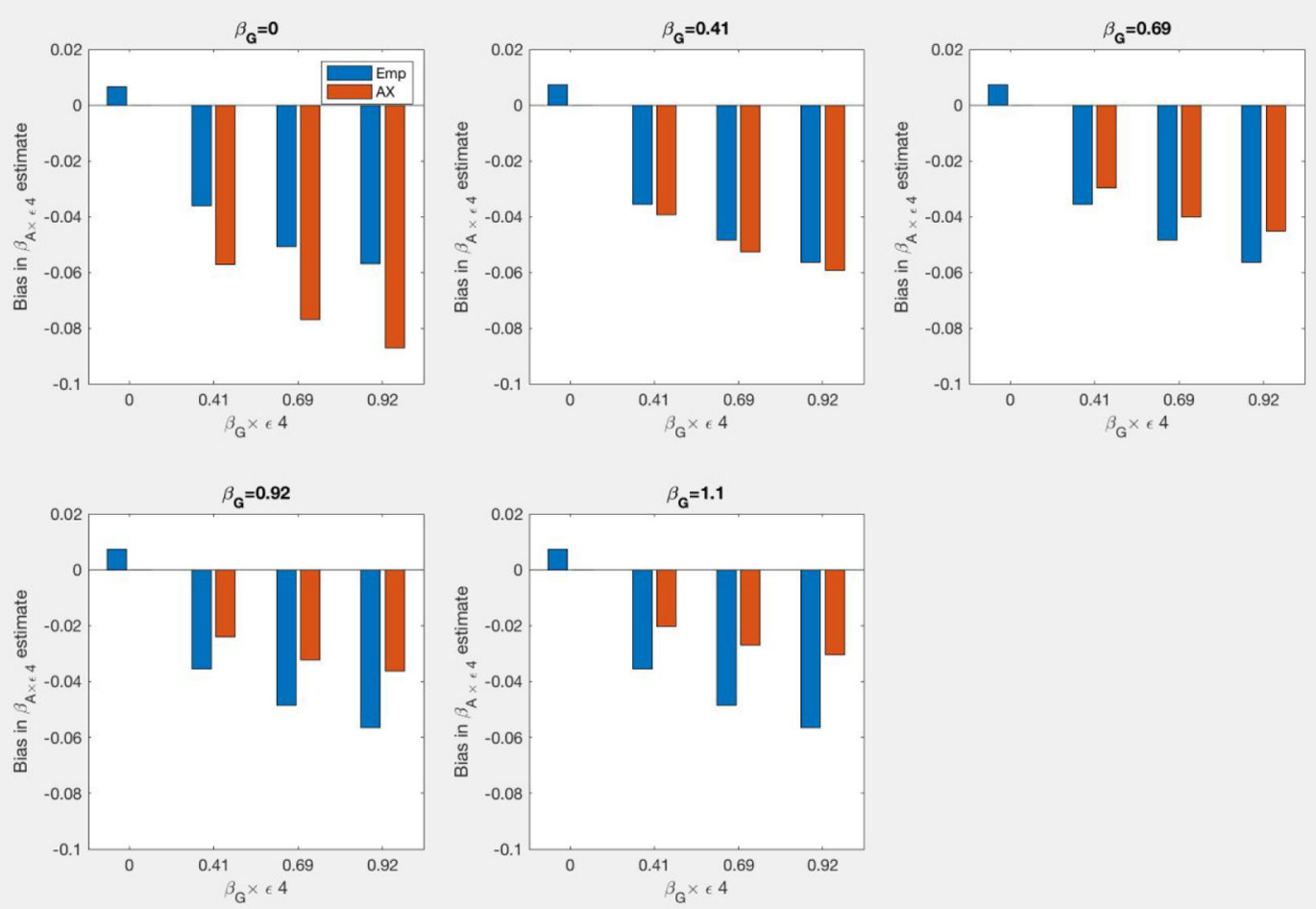

Figure 4.

Empirical bias (Emp) and bias approximated by $(\mathrm{A} 6 \mathrm{~b})(\mathrm{AX})$ in $\beta_{A \times \epsilon 4}$ estimates when the data are generated according to disease states as in (A3)-(A4), but the parameters are estimated in the model (A5). Genotype, age, sex, ApoE $\epsilon 4$ status are simulated to be binary with frequencies $\theta_{G}=0.10, \theta_{A}=0.50, \theta_{S}=0.52, \theta_{\epsilon 4}=0.07$. The pathologically defined disease states are then simulated according to risk model (A3)-(A4)

$$
\beta_{0}=-1, \beta_{S}=\log (0.92)=-0.08, \beta_{\epsilon 4}=\log (8)=2.1, \beta_{A}=\log (2)=0.69 \text {, }
$$

$\beta_{G}=\log (1)=0, \log (1.5)=0.41, \log (2)=0.69, \log (2.5)=0.92, \log (3)=1.1, \beta_{A \times \in 4}=\log (1)=0$, $\log (1.5)=0.41, \log (2)=0.69, \log (2.5)=0.92$,

$\alpha_{0}=-1, \alpha_{S}=\log (0.92), \alpha_{\epsilon 4}=0, \alpha_{A}=\log (2), \alpha_{G}=0, \alpha_{A \times \epsilon 4}=0$. Values of $\beta_{A \times \epsilon 4}$ are along the $\mathrm{x}$-axis and empirical estimates are shown in blue, approximations are in red.. 

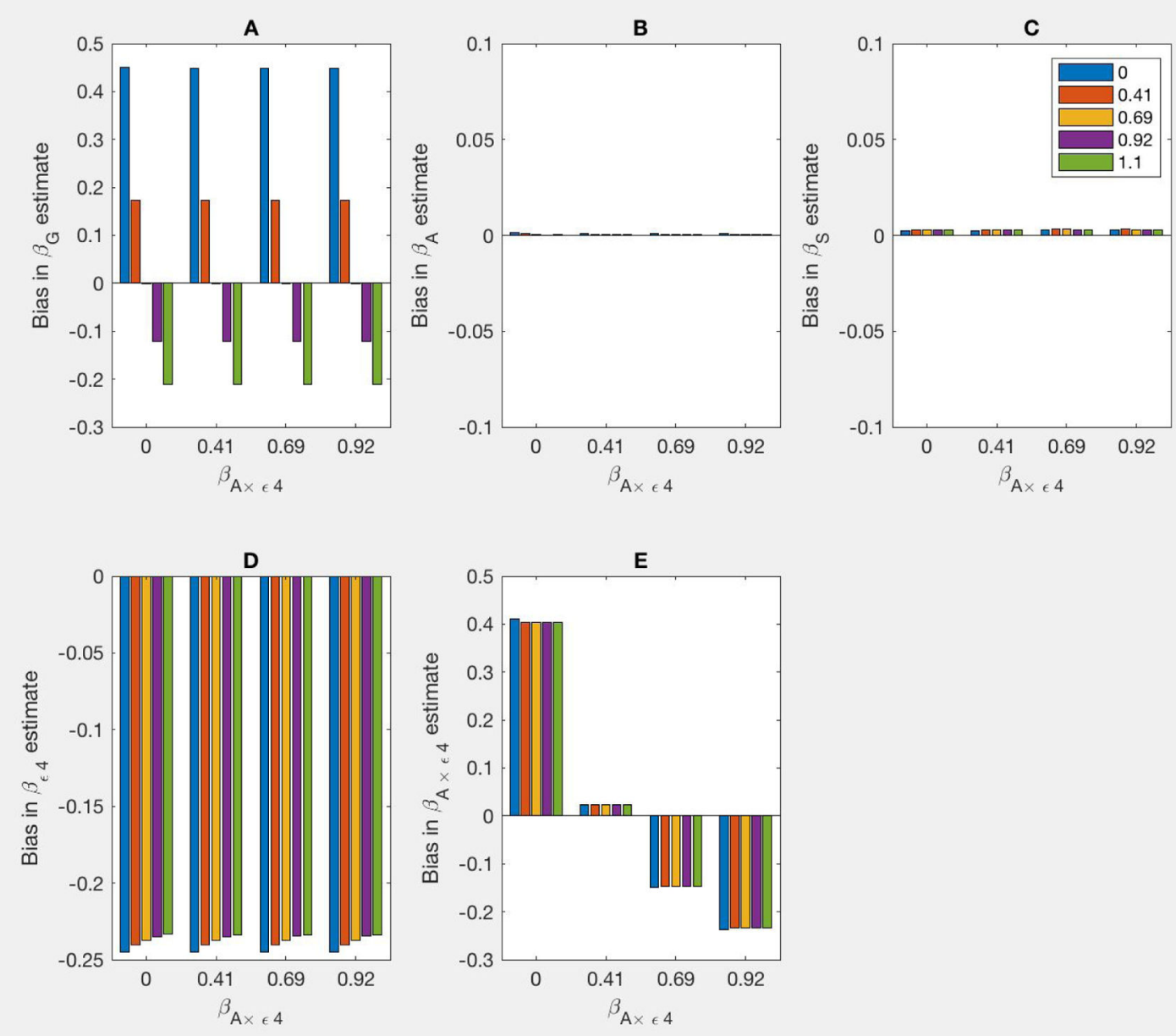

Figure 5 .

Empirical biases in estimates of $\beta_{G}, \beta_{A}, \beta_{S}, \beta_{\epsilon 4}, \beta_{A \times \epsilon 4}$ across 500 datasets with 3,000 cases and 3,000 controls when the disease states are generated as in (A3)-(A4), but the parameters are estimated in the model (A5). Genotype, age, sex, ApoE $\epsilon 4$ status are simulated to be binary with frequencies $\theta_{G}=0.10, \theta_{A}=0.50, \theta_{S}=0.52, \theta_{\epsilon 4}=0.07$. The pathologically defined disease states are then simulated according to risk model (1)-(2) with

$\beta_{0}=1.5, \beta_{S}=\log (0.80), \beta_{\epsilon 4}=\log (8), \beta_{A}=\log (3)$,

$\beta_{G}=\log (1)=0, \log (1.5)=0.41, \log (2)=0.69, \log (2.5)=0.92, \log (3)=1.1, \quad \beta_{A \times \in 4}=\log (1)=0$, $\log (1.5)=0.41, \log (2)=0.69, \log (2.5)=0.92$,

$\alpha_{0}=0.5, \alpha_{S}=\log (0.80), \alpha_{\epsilon 4}=\log (4), \alpha_{A}=\log (3), \alpha_{G}=\log (2), \alpha_{A \times \epsilon 4}=\log (2)$. Values of

$\beta_{A \times \epsilon 4}$ are along the $\mathrm{x}$-axis and values of $\beta_{G}$ are indicated by color. 
Table 1:

False positive rate defined as the proportion of p-values $₫ 0.05$ across 10,000 simulated datasets in the usual logistic regression analyses as an outcome variable (3), when in fact $\beta_{G}=0$ and the data are generated from (1)-(2). We let $\beta_{0}=0.5, \beta_{G}=0, \alpha_{G}=\log (1)=0, \log (1.5)=0.41, \log (2)=0.69$.

\begin{tabular}{|r|c|c|c|}
\hline$a_{G}=$ & $\log (1)=0$ & $\log (1.5)=0.41$ & $\log (2)=0.69$ \\
\hline$n_{0}=n_{1}=3,000$ & 0.052 & 0.72 & 0.99 \\
\hline$n_{0}=n_{1}=10,000$ & 0.048 & 0.79 & 0.99 \\
\hline
\end{tabular}


Table 2:

Empirical estimates of $\beta_{G}$ and approximation (4b). The data are simulated from models (1)-(2) and is estimated using model (3). Empirical estimates are the averages across 500 datasets with 3,000 cases and 3,000 controls. We let $\beta_{0}=\alpha_{0}=0.5$,

$\beta_{G}=\log (1)=0, \log (1.5)=0.41, \log (2)=0.69, \log (2.5)=0.92, \log (3)=1.1, \quad \beta_{\epsilon 4}=\alpha_{\epsilon 4}=\log (8), \quad \alpha_{G}=\log (1)=0$, $\log (2)=0.41, \log (3)=0.69, \log (4)=1.1$.

\begin{tabular}{|c|l|l|l|l|l|}
\hline \multirow{2}{*}{$a_{G}$} & \multicolumn{5}{|c|}{$\beta_{G}$} \\
\cline { 2 - 6 } & $\mathbf{L o g}(\mathbf{1})=\mathbf{0}$ & $\log (\mathbf{1 . 5})=\mathbf{0 . 4 1}$ & $\mathbf{L o g}(\mathbf{2})=\mathbf{0 . 6 9}$ & $\log (\mathbf{2 . 5})=\mathbf{0 . 9 2}$ & $\log (\mathbf{3})=\mathbf{1 . 1}$ \\
\hline $\log (1)=0$ & $0.003, O$ & $0.23,0.22$ & $0.41,0.40$ & $0.57,0.56$ & $0.70,0.69$ \\
\hline $\log (2)=0.41$ & $0.41,0.41$ & $0.57,0.56$ & $0.70,0.69$ & $0.82,0.81$ & $0.92,0.92$ \\
\hline $\log (3)=0.69$ & $0.70,0.69$ & $0.82,0.81$ & $0.93,0.92$ & $1.0,1.0$ & $1.1,1.1$ \\
\hline $\log (4)=1.1$ & $0.93,0.92$ & $1.02,1.01$ & $1.1,1.1$ & $1.2,1.2$ & $1.3,1.3$ \\
\hline
\end{tabular}


Table 3:

Empirical estimates of $\gamma_{G}$ and approximation (8b). The data are simulated from models (5)-(6) and is estimated using model (7). Empirical estimates are the averages across 500 datasets with 3,000 cases and 3,000 controls. We let $\beta_{0}=\alpha_{0}=0.5$,

$\beta_{G}=\log (1), \log (1.5), \log (2), \log (2.5), \log (3), \quad \beta_{\epsilon 4}=\alpha_{\epsilon 4}=\log (8), \quad \alpha_{G}=\log (1), \log (2), \log (3), \log (4)$.

\begin{tabular}{|c|l|l|l|l|l|}
\hline \multirow{2}{*}{$\boldsymbol{a}_{\boldsymbol{G}}$} & \multicolumn{5}{|c|}{$\boldsymbol{\beta}_{\boldsymbol{G}}$} \\
\cline { 2 - 6 } & $\mathbf{L o g}(\mathbf{1})=\mathbf{0}$ & $\log (\mathbf{1 . 5})=\mathbf{0 . 4 1}$ & $\mathbf{L o g}(\mathbf{2})=\mathbf{0 . 6 9}$ & $\log (\mathbf{2 . 5})=\mathbf{0 . 9 2}$ & $\log (\mathbf{3})=\mathbf{1 . 1}$ \\
\hline $\log (1)=0$ & $0.0056, O$ & $0.23,0.22$ & $0.41,0.40$ & $0.57,0.56$ & $0.70,0.69$ \\
\hline $\log (2)=0.41$ & $0.41,0.41$ & $0.57,0.56$ & $0.79,0.69$ & $0.82,0.81$ & $0.92,0.92$ \\
\hline $\log (3)=0.69$ & $0.70,0.69$ & $0.82,0.81$ & $0.92,0.92$ & $1.0,1.0$ & $1.1,1.1$ \\
\hline $\log (4)=1.1$ & $0.92,0.92$ & $1.02,1.01$ & $1,1,1.1$ & $1.2,1.2$ & $1.2,1.3$ \\
\hline
\end{tabular}




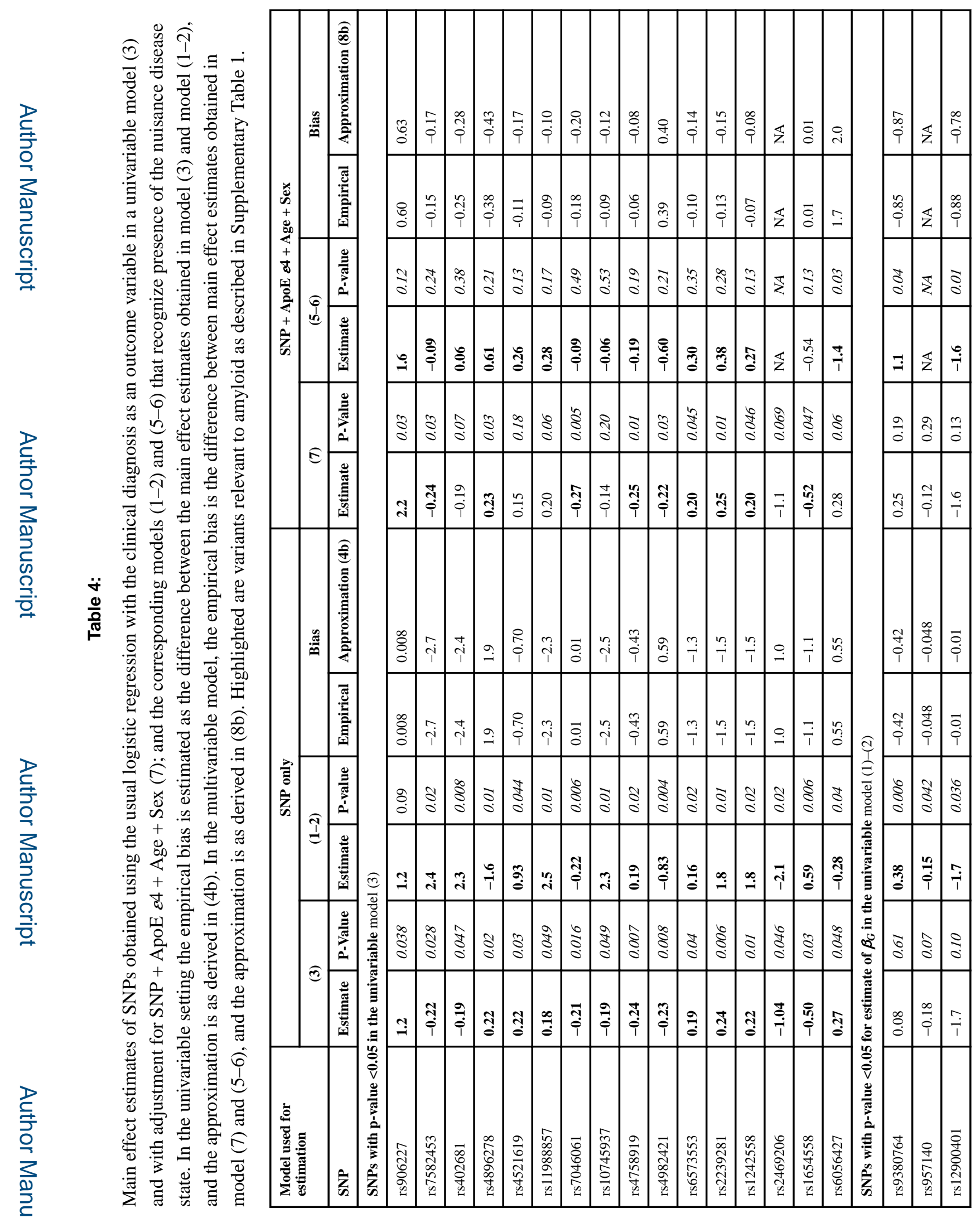

Genet Epidemiol. Author manuscript; available in PMC 2020 July 01. 


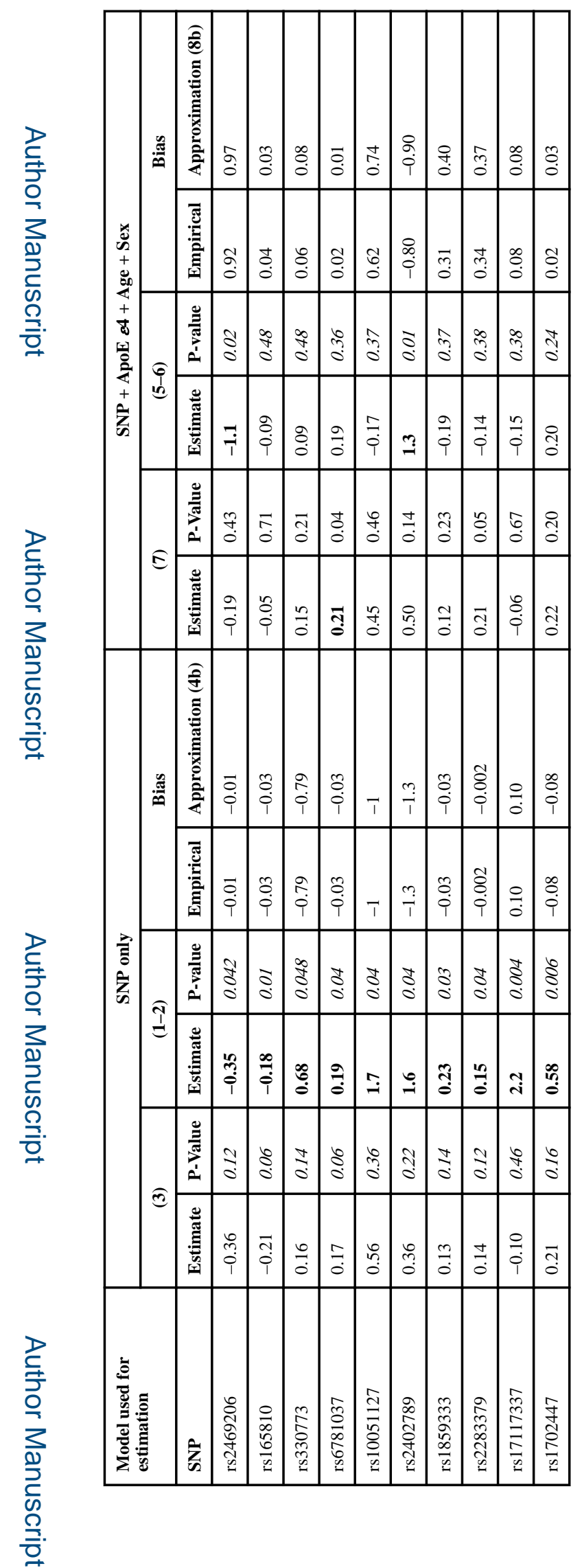

Genet Epidemiol. Author manuscript; available in PMC 2020 July 01. 